\title{
A SUPLEMENTAÇÃO COM ÁCIDO FÓLICO NA GRAVIDEZ: PERCEÇÕES E PRÁTICAS CLÍNICAS NA ÁREA METROPOLITANA DO PORTO
}

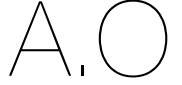

ARTIGO ORIGINAL

1 Unidade de Saúde Pública do Agrupamento de Centros de Saúde Tâmega II - Vale do Sousa Sul da Administração Regional de Saúde do Norte,

Avenida Comendador Abílio Seabra, n. 104 4560-029 Paredes,

Portugal

${ }^{2}$ Centro de Biotecnologia e Química Fina da Escola de Biotecnologia da Universidade Católica Portuguesa,

Rua de Diogo Botelho, ก. 1327

4169-005 Porto, Portugal

${ }^{3}$ EPIUnit - Instituto de Saúde Pública da Universidade do Porto, Rua das Taipas, n. 135 4050-091 Porto, Portugal

${ }^{4}$ Departamento de Biomedicina da Unidade de Bioquímica da Faculdade de Medicina da Universidade do Porto, Alameda Prof. Hernâni Monteiro,

4200-319 Porto, Portugal

${ }^{5}$ Center for Research in Health Technologies and Information Systems da Universidade do Porto, Rua Dr. Plácido da Costa, 4200-450 Porto, Portugal

Endereço para correspondência: Carla Silva Rua da Telheira, n. ${ }^{\circ} 479$ - A 4 , 4250-483 Porto, Portugal carlarib.silva@gmail.com

Histórico do artigo:

Recebido a 1 de agosto de 2019 Aceite a 10 de novembro de 2019

\author{
FOLIC ACID SUPPLEMENTATION IN PREGNANCY: \\ CLINICAL AWARENESS AND PRACTICE IN PORTO REGION
}

Carla Silva ${ }^{1}$; Elisabete Pinto ${ }^{2,3}$; Elisa Keating ${ }^{4,5}$

RESUMO

INTRODUÇÃo: Os suplementos de ácido fólico, comparticipados no mercado português, contendo apenas esta vitamina, contêm uma dose 12,5 vezes maior do que a dose diária recomendada pela Organização Mundial da Saúde, o que faz prever que, em Portugal, as mulheres grávidas façam uma suplementação excessiva em ácido fólico. Por outro lado, o início da suplementação raramente acontece na pré-conceção, como seria desejável.

OBJETIVOS: Conhecer as perceções e as práticas clínicas sobre a prescrição de suplementação com ácido fólico na gravidez e identificar os benefícios e efeitos adversos que os médicos reconhecem nesta suplementação.

METODOLOGIA: Foram realizadas 12 entrevistas semiestruturadas a médicos especialistas em medicina geral e familiar ( $\mathrm{n}=6$ ) ou em obstetrícia ( $n=6)$, em unidades de saúde familiar e hospitais da área metropolitana do Porto, cujo conteúdo, após desgravação, foi codificado em categorias e subcategorias que foram sujeitas a análise qualitativa recorrendo ao software NVIVO 10.0.

RESULTADOS: Foi possível observar que: a) todos os médicos reconhecem a importância da suplementação com ácido fólico na gravidez para a prevenção da ocorrência de malformações do tubo neural, b) há a convicção de que a adesão à toma de ácido fólico é muito elevada ( $n=12)$; c) há unanimidade na descrição de falta de oportunidade para cumprir o início da suplementação antes da conceção e ainda que d) há um razoável desconhecimento em relação à dose de ácido fólico prescrita $(n=7)$.

CONCLUSÕES: Considerando os estudos recentes que sugerem efeitos adversos da suplementação com ácido fólico em doses excessivas e temporalmente desadequadas, este estudo evidencia a pertinência de um esforço conjunto nacional para a homogeneização das práticas clínicas na prescrição de suplementação com ácido fólico na gravidez.

\section{PALAVRAS-CHAVE}

Ácido fólico, Gravidez, Medicina geral e familiar, Obstetrícia, Período de suplementação

ABSTRACT

INTRODUCTION: Folic acid supplements reimbursed by the Portuguese Government that contain solely this vitamin have a dose 12.5 times higher than the daily dose recommended by the World Health Organization. So, it is expectable that Portuguese pregnant women are taking excessive folic acid supplementation. On the other hand, the beginning of supplementation rarely occurred in the preconception, as desirable.

OBJECTIVES: To explore the perceptions and clinical practices concerning the prescription of folic acid supplementation in pregnancy and to identify the beneficial and adverse effects acknowledged by the physicians regarding this supplementation. METHODOLOGY: Twelve semi-structured interviews were performed (Specialists in Family Medicine $(\mathrm{n}=6$ ) or specialists in Obstetrics $(\mathrm{n}=6)$ working at primary care units and hospitals of the Grande Porto region) and the content was codified in categories and sub-categories and analysed with NVIVO 10.0 software.

RESULTS: We could observe that: a) all the physicians acknowledged the relevance of folic acid supplementation in pregnancy for the prevention of neural tube defects, b) the physicians recognized that compliance by pregnant women to folic acid intake is very high $(n=12) ; c)$ all the physicians acknowledge the difficulty to comply with the ideal timing for folic acid supplementation before conception; d) the ideal folic acid dose is largely unknown by the physicians $(n=7)$.

CONCLUSIONS: Considering recent studies that suggest adverse effects of excessive and chronologically unsuitable folic acid supplementation, this study evidences that there should be a national joint effort towards the homogenization of clinical practices regarding the prescription of folic acid supplementation in pregnancy.

\section{KEYWORDS}

Folic acid, Pregnancy, Family medicine, Obstetrics, Supplementation period 


\section{INTRODUÇÃO}

Desde os anos 90 do século passado, quando apareceu evidência contundente acerca do efeito do ácido fólico (AF) na prevenção das malformações do tubo neural $(1,2)$, surgiram diferentes estratégias para a suplementação e a fortificação de alimentos com esta vitamina. Estas estratégias incluíam a suplementação massiva das mulheres na periconceção, assumindo-se que a prevalência de inadequação em folato era elevada e que, pelo facto de se tratar de uma vitamina hidrossolúvel, qualquer excesso no aporte originaria a sua excreção, sem qualquer prejuízo. Efetivamente, a prevalência de inadequação em folato em mulheres portuguesas era, num estudo publicado em 2009, de 58,2\% no ano prévio à conceção e de 90,8\% durante a gravidez (3). Atualmente, a Organização Mundial da Saúde (OMS) preconiza a suplementação diária com 0,4 mg de AF, durante a gravidez, a iniciar o mais precocemente possível. No caso de mulheres com risco acrescido de malformações fetais (ou seja, grávidas com filho anterior com defeito do tubo neural ou com história familiar desta situação ou ainda mulheres com doenças ou sob terapêutica associadas a diminuição da biodisponibilidade de AF) a recomendação da OMS é de $5 \mathrm{mg}$ de AF por dia $(4,5)$. Em qualquer caso, é assumido que a proteção em relação às malformações do tubo neural apenas é eficaz quando a suplementação ocorre nas primeiras quatro semanas de gestação (6). Paralelamente, têm surgido estudos que suportam o papel nefasto da suplementação com ácido fólico em doses excessivas (7-12). Sabe-se que a ingestão de doses maiores do que 0,4 mg/dia de AF origina o aparecimento de AF não metabolizado na corrente sanguínea materna e fetal, facto que tem sido sugerido como responsável pela alteração da regulação génica, com consequências na programação fetal de doenças, tais como asma, insulinorresistência, alguns tipos de cancro ou autismo $(15,16)$. Em Portugal, através da aplicação da prescrição eletrónica médica (PEM) do Sistema Nacional de Saúde, a dose de 0,4 mg por comprimido encontra-se apenas disponível em associação com outras vitaminas e/ou minerais, não sendo estes suplementos comparticipados pelo Sistema Nacional de Saúde. Os suplementos comparticipados disponíveis no mercado português contendo apenas esta vitamina contêm 5 mg por comprimido, ou seja, uma dose 12,5 vezes maior de que a dose diária recomendada pela OMS. Prevê-se, pois, que a esmagadora maioria das mulheres grávidas faça uma suplementação excessiva em AF (17). Adicionalmente, é também previsível que algumas mulheres iniciem esta suplementação somente após terem tido conhecimento da sua gravidez (17), o que na maioria dos casos ocorre após o período crítico para a prevenção das malformações do tubo neural.

A suplementação de acordo com a evidência atual dependerá de muitos fatores, nomeadamente o conhecimento sobre ácido fólico dos profissionais de saúde que prescrevem estes suplementos às futuras mães e, consequentemente, das suas práticas clínicas. A caracterização destes conhecimentos e práticas clínicas é a grande motivação deste estudo, uma vez que se desconhece a dimensão desta realidade. Optou-se assim pela realização de um estudo qualitativo tendo como princípio o facto de que as práticas quotidianas dos profissionais não decorrem apenas das orientações teóricas mas são resultado de forças, fatores, estruturas internas e externas que atuam sobre as pessoas, gerando determinados resultados. A abordagem qualitativa permite, então, produzir informação com base na experiência vivida dos seres humanos, que pode ser de extrema importância no contexto da prática médica e na interpretação de práticas que já estão enraizadas há várias décadas, como acontece com o AF durante a gravidez (18)

\section{OBJETIVOS}

Este estudo tem como objetivos concretos: a) conhecer as perceções e as práticas clínicas de médicos no que respeita à prescrição de suplementação com AF na gravidez, nomeadamente no que diz respeito à dose e ao período de suplementação, bem como b) identificar os benefícios e efeitos adversos que os profissionais reconhecem nesta suplementação. Este estudo pretende responder à questão de investigação "Qual o nível de conhecimento e quais as práticas clínicas de prescrição de suplementação com AF na gravidez, em Portugal?"

\section{METODOLOGIA}

Este estudo qualitativo baseia-se na informação recolhida em entrevistas semiestruturadas, realizadas a médicos das duas especialidades que mais frequentemente são responsáveis pela vigilância de mulheres grávidas: especialistas em medicina geral e familiar (MGF) e especialistas em obstetrícia (OBS). Optou-se por realizar estas entrevistas num hospital universitário - Centro Hospitalar de S. João, E.P.E. (Porto) - e em três unidades de saúde familiares (USF) do Agrupamento de Centros de Saúde (ACeS) de Gondomar, situado na área de referenciação desse hospital, bem como num hospital não universitário - Centro Hospitalar Tâmega e Sousa, E.P.E. - Hospital Padre Américo, Vale do Sousa (Penafiel) - e numa USF de grande dimensão do ACeS Tâmega II - Vale Sousa Sul. O estudo foi aprovado pela Comissão de Ética do Centro Hospitalar de S. João, E.P.E. e pela Comissão de Ética da Unidade Hospital Padre Américo e teve o aval dos Diretores de todos os Serviços envolvidos. Os objetivos e procedimentos do estudo foram devidamente explicados a todos os potenciais participantes, que consentiram livremente participar, não se tendo verificado qualquer recusa.

Foi inicialmente decidido pelas autoras que seriam entrevistados três OBS de cada hospital envolvido, bem como três MGF de cada ACeS, na tentativa de capturar maior diversidade de perceções e práticas. No total foram realizadas 12 entrevistas presenciais no local de trabalho dos profissionais. O objetivo do estudo foi claramente explicado, com base num guião previamente preparado que garantiu que a explicação foi a mesma para todos os entrevistados. No início da entrevista foi solicitada autorização para gravação áudio. As gravações foram destruídas após desgravação integral. Os materiais resultantes da entrevista foram anonimizados e foi assim mantida a total confidencialidade dos dados recolhidos. As entrevistas decorreram entre outubro e novembro de 2014. Cada entrevista teve uma duração aproximada de 15 minutos. O guião das entrevistas (anexo I) foi concebido pelas autoras. No início de cada entrevista foi ainda perguntado a cada profissional quantos anos de especialidade médica possuía. Todas as entrevistas foram integralmente transcritas, tendo posteriormente sido sujeitas a leitura flutuante para identificação das porções significativas dos dados e atribuição de categorias/ subcategorias. As categorias criadas foram as seguintes: a) período ideal para a suplementação, b) dosagem aconselhada, c) fundamentação para a sua realização, d) formas de prescrição dos suplementos (isolados ou em associação com outras vitaminas e/ou minerais), e) fontes de informação utilizadas pelos profissionais para se informarem sobre esta temática, f) identificação de potenciais efeitos. Tendo-se utilizado uma abordagem indutiva na análise dos dados, este sistema de categorias foi-se, naturalmente, complexificando à medida que as entrevistas foram codificadas, tendo-se criado diferentes subcategorias. Três das 12 entrevistas foram independentemente codificadas por duas das autoras, tendo-se posteriormente procedido à avaliação da concordância das codificações, que foi considerada 
elevada. As 12 entrevistas foram classificadas segundo a especialidade médica do entrevistado, a classe de cuidados de saúde prestados (primários versus diferenciados), a localização geográfica do local de trabalho, bem como segundo a duração da sua experiência profissional na especialidade atual, categorizada em intervalos de 10 anos. Os padrões e regularidades dos dados foram notificados pelas respetivas frequências com que apareciam nas entrevistas. Contudo, foi também dado o devido destaque a ocorrências únicas de perceções ou práticas. As expressões mais ilustrativas das perceções e práticas dos médicos em relação à suplementação com AF no contexto da gravidez foram selecionadas para apresentação neste trabalho. A análise foi validada pelas três autoras e as dúvidas foram resolvidas por consenso, após discussão conjunta. Foram realizadas matrizes de relação entre diferentes categorias para uma interpretação mais aprofundada dos dados, nomeadamente a observação de quaisquer padrões nas informações obtidas de acordo com os atributos dos entrevistados. A análise de conteúdo foi realizada com recurso ao software NVIVO 10.0.

\section{RESULTADOS}

Perceção Clínica sobre a Importância da Suplementação da Grávida com Ácido Fólico

A maioria dos médicos entrevistados considera que a suplementação com AF na periconceção é importante em todas as mulheres. Dos 12 entrevistados, 9 referem que a suplementação é importante: "sempre" ou "em todas as mulheres" e três médicos, embora não respondam diretamente à questão, assumem como adquirido que esta suplementação deve ser feita em todas as mulheres que planeiam uma gravidez e, de facto, todos dizem prescrever esta vitamina nas mulheres grávidas, assim que as contactam pela primeira vez e, sempre que possível, naquelas que planeiam uma gravidez. Dos 12 entrevistados, três (todos obstetras) referem adicionalmente que há grupos específicos de mulheres com indicação adicional para suplementação com AF, sendo referidas como exemplo as mulheres com mutações na metilenotetrahidrofolato redutase ou com medicação para a epilepsia. Quando questionados sobre o objetivo da suplementação com AF, a prevenção das malformações do tubo neural é o motivo mais referido, sendo que apenas dois dos entrevistados referem genericamente malformações ou auxílio da embriogénese, não especificando a importância na formação do tubo neural. Para além de reconhecerem a importância da suplementação com AF na prevenção das malformações do tubo neural, alguns médicos consideram também as anemias, a prevenção de malformações fetais ou cardiopatias ou medicação materna específica, como razões para a prescrição de AF. Verificou-se que o tempo de serviço não parece afetar a opinião clínica sobre a importância da suplementação com AF na gravidez. Verificou-se ainda que os OBS apontam mais frequentemente as anemias e a prevenção de cardiopatias como fundamentação para a suplementação com AF, quando comparados com os MGF.

\section{Perceção Clínica sobre os Efeitos Adversos da Suplementação da Grávida com Ácido Fólico}

Quando questionados sobre possíveis efeitos adversos da suplementação da mulher com AF, 11 médicos afirmaram não haver ou nunca terem constatado a ocorrência de efeitos adversos para a mulher, para o bebé ou para ambos. Um dos médicos entrevistados refere ter "conhecimento (...) de um estudo feito por nutricionistas que levantava (...) [a questão de] aumento (...) do risco de cancro da mama em mulheres que faziam suplementação com quantidades excessivas de AF durante longos períodos de tempo", mas completou afirmando que "(...) [o estudo] não era muito conclusivo".

\section{Perceção Clínica sobre as Janelas Temporais Ótimas para a Suplementação com Ácido Fólico}

Muitos dos clínicos referiram claramente existir uma distinção entre o que seria o período ideal e o período em que efetivamente conseguem prescrever a suplementação (timing real), que é dependente do momento em que as mulheres os procuram, frequentemente após gravidez confirmada. A maioria dos médicos considera que o período, pré-concecional, bem como o primeiro trimestre de gravidez, são as janelas temporais ideais para a suplementação com AF. Na maioria dos casos, são estes os períodos reais de prescrição. No entanto, foi evidente no discurso dos clínicos a dificuldade em conseguir a suplementação na pré-conceção. A este respeito um médico refere: "Essa suplementação deve ser iniciada na pré-conceção, idealmente", um outro responde: “(...) pelo menos, um mês antes [da conceção], [a suplementação] (...) deveria [iniciar], embora isso nem sempre é possível.". No que respeita ao término da suplementação, verificou-se muito mais heterogeneidade de práticas. Curiosamente, embora alguns dos médicos reconheçam que a suplementação deveria ser mantida apenas durante o primeiro trimestre, a prática clínica real não corresponde a esta opinião, havendo cerca de metade dos médicos que afirmam manter a suplementação até ao final da gravidez com algumas referências para a manutenção durante o aleitamento. Um dos médicos afirma que: "Em princípio mantenho 5 mg [de AF] até ao final da gravidez."; um outro refere: "Faço sempre mais ou menos quatro meses antes de programar a gravidez, e depois durante toda a gravidez, até mesmo a amamentar (...)", prática corroborada por um outro médico que diz: "(...) mantenho durante a gravidez toda e aleitamento, também.". Quando se perguntou qual a fundamentação da opinião clínica sobre as janelas temporais ótimas para suplementação com AF, a evidência científica e a experiência clínica foram as justificações mais apontadas ( $n=8$ e $n=6$, respetivamente), com uma baixa frequência de referência concreta à circular normativa da Direção-Geral da Saúde relativa a esta temática $(n=3)$. Embora a localidade de exercício de funções não pareça influenciar a perceção clínica sobre as janelas temporais ideais de suplementação com AF, verificou-se que os OBS, mais do que os MGF, apontam a pré-conceção e o primeiro trimestre como períodos ideais. Paralelamente, nenhum MGF aponta toda a gravidez e o aleitamento como períodos ideais ótimos de suplementação, o mesmo não acontecendo com os OBS. Relativamente às janelas temporais de suplementação efetivamente praticadas (timing real), observa-se que os clínicos com experiência de serviço entre 10 e 20 anos são aqueles que referem nunca prescrever a suplementação com AF após o primeiro trimestre de gravidez. É também importante realçar que há um maior número de MGF que efetivamente preconizam a suplementação com AF até ao final da gravidez, quando comparado com os OBS. A localidade de exercício de funções não parece afetar os períodos de suplementação efetivamente recomendados pelos clínicos.

\section{Dose de Ácido Fólico Prescrita}

A pergunta sobre a dose de AF que é habitualmente prescrita às mulheres no período periconcecional ou durante a gravidez foi aquela que gerou mais hesitações. De facto, 7 dos entrevistados não sabiam ou revelavam uma clara confusão sobre a dose de AF existente no suplemento que prescreviam, como ilustrado pelos seguintes trechos: "Não tenho ideia da dosagem... agora não tenho ideia da dosagem dos comprimidos, mas normalmente é um comprimido."; "Temos [para prescrição] o Folici ${ }^{\circledR}$, que é 400 se não estou em erro (...)”. A esmagadora maioria dos médicos referiu que prescreve Folicil ${ }^{\circledR}$, um dos 
suplementos disponíveis no mercado português, que contém $5 \mathrm{mg}$ de AF por comprimido e/ou referiu especificamente a dose de $5 \mathrm{mg}$ por dia. Contudo, embora com baixa frequência, registou-se a referência a outras doses como 0,4 mg e $1 \mathrm{mg}$. A dose de 0,4 mg foi referida como a dose ideal ou como a dose existente em multivitamínicos. Dos 12, apenas um médico mostrou colocar em prática a preocupação sobre o excesso de AF, referindo: "(...) digo (...) [à grávida que], não precisa de tomar um comprimido de $5 \mathrm{mg}$ todos os dias. Às vezes opto por lhes dizer... tome 3 ou 4 comprimidos por semana, ou tome dia sim, dia não, enfim a dose não é preciso ser exatamente aquela que vem no comprimido, que é muito superior à recomendada (...)".

\section{Prescrição de Ácido Fólico em Multivitamínicos ou em Associação com Outros Micronutrientes}

A maioria dos médicos entrevistados referiu prescrever micronutrientes além do $A F$, sendo que o iodo é o micronutriente mais prescrito, normalmente numa formulação independente do AF. Apenas três médicos referiram prescrever ferro, para além do $A F$, em formulações independentes ou em associação. A alusão a multivitamínicos foi muito menos frequente, sendo feita em função da alimentação praticada pela mulher, como é visível nos seguintes excertos: "[A decisão de prescrição de multivitamínicos] tem muito a ver, muito, com o tipo de alimentação [da grávida] (...)"; "Depende do tipo de alimentação que a grávida tem e do meio em que vive.".

\section{DISCUSSÃO DOS RESULTADOS}

É inequívoca a relevância da suplementação com AF durante a gravidez, por estar solidamente demonstrado que esta suplementação previne a ocorrência e a recorrência de defeitos do tubo neural na descendência $(1,2)$. Em Portugal, a Direção-Geral da Saúde, através da circular normativa N. ${ }^{0}$ : 02/DSMIA de 16/01/06, recomenda a suplementação com ácido fólico "a iniciar pelo menos dois meses antes da interrupção do método contracetivo", e apenas no final de 2015, passou a ser mencionada a dosagem em que esta deve ocorrer. Esta medida deveria, naturalmente, ser acompanhada da disponibilização no mercado português de comprimidos contendo somente 0,4 mg de AF. Neste contexto, foi objetivo principal deste estudo conhecer as perceções e as práticas clínicas no que respeita à prescrição de suplementação com AF no contexto de uma gravidez. Este estudo constitui uma primeira aproximação a esta problemática, não sendo garantido que espelha a opinião de todos os profissionais dos grupos profissionais versados. Contudo, o facto de se ter entrevistado médicos de especialidades diferentes, a trabalhar em contextos diferentes (Cuidados de Saúde Primários e Cuidados Especializados Hospitalares) contribui certamente para a diversidade das opiniões que foram encontradas. Em relação a algumas temáticas, como por exemplo a importância da realização de suplementação com AF por todas as mulheres na periconceção, foi obtida elevada consistência nas respostas o que nos leva a afirmar que foi esgotada a variabilidade de respostas possíveis com as entrevistas realizadas. Contudo, um estudo anterior baseado na coorte de nascimento Geração XXI mostrou que, numa subamostra de 249 grávidas, $56,6 \%$ referiram ter planeado a gravidez, mas somente $18,6 \%$ tinha realizado suplementação com AF na pré-conceção, não cumprindo portanto a prática recomendada (3). Não obstante a subjetividade inerente ao conceito de planeamento de gravidez, em particular na perceção das mulheres, estes números indicam que há espaço para informar melhor as mulheres em idade fértil. Assim, quisemos explorar os conhecimentos e práticas de alguns médicos acerca da temática. De um modo geral, no presente estudo observou-se que todos os médicos reconhecem a importância do AF no contexto de uma gravidez, sendo praticamente unânimes em considerar que este previne a ocorrência de malformações do tubo neural e não têm experiência da ocorrência de efeitos adversos. Contudo, apontam, por vezes, outras indicações para a realização de suplementação para as quais a evidência é menos consistente (19). Quanto às janelas temporais ótimas de suplementação, muitos médicos defendem como início ideal da suplementação a pré-conceção e/ou primeiro trimestre de gravidez, mas no que respeita ao término dessa janela de maximização do benefício existe uma maior diversidade de opiniões. Foi possível perceber unanimidade na dificuldade de implementação deste timing ideal de suplementação, por falta de oportunidade, já que, como é do conhecimento generalizado, uma grande parte das mulheres não realiza consulta de planeamento da gravidez, recorrendo aos serviços de saúde apenas quando sabem que estão grávidas. É importante referir que esta falta de oportunidade não deverá justificar o prolongamento da suplementação para além do período ideal preconizado, embora esta ideia tenha ficado subjacente em algumas considerações tecidas pelos médicos, no presente estudo.

Efetivamente, a falta de informação sobre os potenciais riscos de uma suplementação excessiva em AF, pode estar na base da decisão clínica da manutenção da suplementação ao longo da gravidez, mesmo sem que este prolongamento esteja previsto nas orientações ou normas clínicas. Como referido acima e revisto anteriormente pelo nosso grupo, este excesso poderá acarretar riscos para a mãe e para o filho (15), além de poder interferir com os níveis de vitamina B12, acarretando consequências importantes para a mulher (20).

De uma forma muito consistente, observou-se desconhecimento em relação à dose de AF prescrita, muitas vezes com a assunção, errónea, de que as formulações disponíveis nas farmácias portuguesas e indicadas para o efeito possuem a dose adequada. Este parece ser, por isso, um ponto crucial que merece maior esclarecimento entre os clínicos.

Por outro lado, foi interessante perceber que a prescrição de multivitamínicos foi frequentemente referida como sendo realizada com ajuste ao tipo de alimentação da grávida, sem contudo se perceber a existência de uma recomendação generalizada destas formulações. Ainda com respeito a esta questão observou-se que a maioria dos médicos prescreve também iodo à mulher grávida, revelando grande adesão à orientação técnica 011/2013 da Direção-Geral da Saúde. Não deixa de ser curioso que apenas três médicos tenham feito alusão à suplementação com ferro. No estudo realizado por Pinto et al. (3) a suplementação com este mineral ocorreu em cerca de $76 \%$ das grávidas a partir do segundo trimestre de gravidez, ultrapassando os $40 \%$ já no primeiro trimestre. É fortemente recomendado que se explore igualmente o conhecimento dos enfermeiros sobre este mesmo tema, nomeadamente por atualmente surgir a figura do enfermeiro de família que se pretende que tenha um contacto muito próximo com as pessoas, tendo um papel decisivo na educação para a saúde.

Apesar de este estudo ter sido conduzido em 2014/2015, até ao momento não existiu por parte da Direção-Geral da Saúde nenhuma revogação da circular normativa N. ${ }^{\circ}$ : 02/DSMIA de 16/01/06 nem foi criado nenhum grupo de trabalho, à semelhança do que foi criado pela Direção-Geral da Saúde em novembro de 2018 sobre a suplementação em iodo da criança e da grávida, com o intuito de rever as orientações de suplementação bem como de definir normas de monitorização. Este facto demonstra uma evidente lacuna nesta área e demonstra a pertinência e atualidade do tema estudado. Adicionalmente, com base em investigação subsequente ainda não publicada, os autores verificaram que a posição dos clínicos se mantém inalterada. 


\section{CONCLUSÕES}

Em suma, este estudo descreve perceções e práticas clínicas sobre a prescrição de suplementação com AF na gravidez, evidenciando por um lado o reconhecimento generalizado da importância desta vitamina na gravidez mas, por outro, que há inconsistência nas perceções clínicas no que respeita aos períodos ideais de suplementação, em particular a altura ideal de paragem de suplementação, e no que respeita à dose de AF a suplementar. Por estes motivos, e dado os estudos existentes sobre os potenciais efeitos adversos da suplementação com doses excessivas e/ou temporalmente desadequadas, este estudo evidencia a pertinência de um esforço conjunto nacional para a homogeneização de práticas clínicas concretas no que diz respeito à prescrição de suplementação com AF na gravidez, sugerindo-se a revisão da circular normativa em vigor (N.': 02/DSMIA de 16/01/06) com retificação da dosagem de AF a suplementar e a introdução no mercado português de um medicamento comparticipado contendo apenas AF com a dosagem recomendada de $0,4 \mathrm{mg}$.

\section{AGRADECIMENTOS}

Este artigo foi financiado por fundos nacionais através da FCT Fundação para a Ciência e Tecnologia e CINTESIS, Unidade de I\&D (referência UID/IC/5255/2019).

\section{REFERÊNCIAS BIBLIOGRÁFICAS}

1. Wald $\mathrm{N}$ and Sneddon J. Prevention of neural tube defects: results of the Medical Research Council Vitamin Study. MRC Vitamin Study Research Group. Lancet,1991. 338(8760):131-7. doi.org/10.1016/0140-6736(91)90133-A.

2. Czeizel $A E$ and Dudas I. Prevention of the first occurrence of neural-tube defects by periconceptional vitamin supplementation. N Engl J Med, 1992. 327(26):1832-5. doi: 10.1056/NEJM199212243272602.

3. Pinto E, Barros H, Santos Silva I. Dietary intake and nutritional adequacy prior to conception and during pregnancy: a follow-up study in the north of Portugal. Public Health Nutr, 2009. 12(7): 922-31. doi: 10.1017/S1368980008003595.

4. WHO, Guideline: Daily iron and folic acid supplementation in pregnant women. Geneva. 2012, World Health Organization.

5. Direcção-Geral da Saúde. Programa Nacional para a Vigilância da Gravidez de Baixo Risco (2015). ISBN 9789726752332.

6. Mrc Vitamin StudyRresearch Group. Prevention of neural tube defects: Results of the Medical Research Council Vitamin Study. Lancet 338, 131-137, 1991. doi. org/10.1016/0140-6736(91)90133-A.

7. Keating E, Correia-Branco A, Araujo JR, Meireles M, Fernandes R, Guardao L, et al. Excess perigestational folic acid exposure induces metabolic dysfunction in post-natal life. J Endocrinol, 2015. 224(3):245-59. doi: 10.1530/JOE-14-0448.

8. Roy S, Kale A, Dangat K, Sable P, Kulkarni A, and Joshi S. Maternal micronutrients (folic acid and vitamin $B(12)$ ) and omega 3 fatty acids: implications for neurodevelopmental risk in the rat offspring. Brain Dev, 2012. 34(1):64-71. doi: 10.1016/j.braindev.2011.01.002.

9. Mikael LG, Deng L, Paul L, Selhub J, and Rozen R. Moderately high intake of folic acid has a negative impact on mouse embryonic development. Birth Defects Res A Clin Mol Teratol, 2013. 97(1):47-52. doi: 10.1002/bdra.23092.

10. Plumptre L, Masih S, Ly A, Aufreiter S, Sohn K, Croxford R. et al. High concentrations of folate and unmetabolized folic acid in a cohort of pregnant Canadian women and umbilical cord blood. The American Journal of Clinical Nutrition, Volume 102, Issue 4, October 2015, 848-857.

11. Chu D, Li L, Jiang Y, Tan J, Ji J, Zhang Y, et al. Excess Folic Acid Supplementation Before and During Pregnancy and Lactation Activates Fos Gene Expression and Alters Behaviors in Male Mouse Offspring. Front Neurosci. 2019. doi:10.3389/fnins.2019.00313. 12. Tojal A, Neves C, Veiga H, Ferreira S, Rodrigues I, Martel F. et al. Perigestational high folic acid: impact on offspring's peripheral metabolic response. Food Funct. 2019 Nov 1; 10(11):7216-7226. doi: 10.1039/c9fo01807g.
13. Yang NV, Pannia E, Chatterjee D, Kubant R, Ho M, Hammoud R, et al. Gestational folic acid content alters the development and function of hypothalamic food intake regulating neurons in Wistar rat offspring post-weaning.Nutr Neurosci. 2018 May 30:112. doi: 10.1080/1028415X.2018.1479628.

14. Huang, Y. He, X. Sun, Y. He Y. Li and C. Sun, Maternal high folic acid supplement promotes glucose intolerance and insulin resistance in male mouse offspring fed a highfat diet, Int. J. Mol. Sci. 2014, 15, 6298-6313. doi: 10.3390/ijms15046298.

15. Silva $C$, Keating E, Pinto E. The impact of folic acid supplementation on gestational and long term health: Critical temporal windows, benefits and risks. Porto Biomed. 2017;2(6):315-32. doi.org/10.1016/j.pbj.2017.05.006.

16. Whitrow MJ, Moore VM, Rumbold AR, and Davies MJ. Effect of supplemental folic acid in pregnancy on childhood asthma: a prospective birth cohort study. Am J Epidemiol, 2009. 170(12):1486-93. doi: 10.1093/aje/kwp315.

17. Instituto Nacional de Saúde Doutor Ricardo Jorge. Prevenção primária dos defeitos do tubo neural - adesão à toma de ácido fólico: resultados preliminares da Rede Médicos- Lisboa: Boletim epidemiológico n²0, $2^{\text {a }}$ série. INSA, 2007.(acedido em 10 de novembro de 2019), disponível em http://hdl.handle.net/10400.18/4872.

18. Cleland JA. The qualitative orientation in medical education research. Korean J Med Educ. 2017;29(2):61-71. doi:10.3946/kjme.2017.53.

19. Chmielewska A, Dziechciarz P, Gieruszczak-Białek D, Horvath A, Pieścik-Lech M, Ruszczyński M, et al. H.Effects of prenatal and/or postnatal supplementation with iron, PUFA or folic acid on neurodevelopment: Update. British Journal of_Nutrition. 2019. 122(S1). doi:10.1017/S0007114514004243.

20. Paul L, Selhub J. Interaction between excess folate and low vitamin B12 status. Vol. 53, Molecular Aspects of Medicine. Elsevier Ltd; 2017. p. 43-7. doi: 10.1016/j. mam.2016.11.004. 


\section{ANEXO 1}

\section{GUIÃO DA ENTREVISTA}

Através das perguntas que seguidamente lhe colocarei, gostaríamos de saber a sua opinião sobre a suplementação com ácido fólico no contexto de uma gravidez.

1. Em que situações considera que deve ser prescrito o ácido fólico a uma mulher na periconceção?

2. Que efeitos considera que a suplementação com ácido fólico poderá ter para a mulher e/ou a feto/criança?

3. Considera a existência de períodos específicos (durante todo o período que se estende da pré-conceção até ao final do aleitamento) em que suplementação com ácido fólico pode ter os seus efeitos otimizados? (se sim, pedir para indicar quais as janelas temporais)

4. Em que base assenta a sua opinião: na sua experiência clínica, na evidência científica ou em qualquer outro motivo? (qual?)

5. Habitualmente qual a dosagem de ácido fólico que prescreve a uma mulher na periconceção e/ou gravidez?

6. Costuma prescrever suplementos contendo somente ácido fólico, multivitamínicos contendo ácido fólico, ambos ou a sua forma de prescrever é personalizada na grávida?

7. Se prescreve personalizadamente, explique sucintamente como decide qual a suplementação a instituir. 